\title{
Performance of commercial laying hen genotypes on free range and organic farms in Switzerland, France and The Netherlands
}

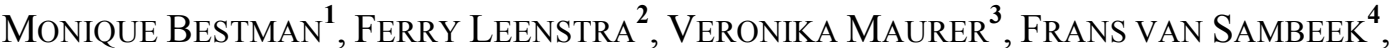 \\ ESTHER ZELTNER ${ }^{3}$, BERRY REUVEKAMP ${ }^{2}$, ZIVILE AMSLER-KEPALAITE ${ }^{3}$, \\ FABIEN GALEA $^{4}$ AND THEA VAN NIEKERK ${ }^{2}$ \\ ${ }^{1}$ Louis Bolk Institute, The Netherlands, www.louisbolk.nl, m.bestman@louisbolk.nl \\ ${ }^{2}$ Wageningen UR Livestock Research, The Netherlands \\ ${ }^{3}$ Research Institute of Organic Agriculture (FiBL), Switzerland \\ ${ }^{4}$ Institut de Sélection Animale (ISA), The Netherlands/France \\ www.lowinputbreeds.org
}

\begin{abstract}
Free range and organic systems provide different circumstances for laying hens than closed houses or cages, where most hens are selected in. An enquiry and farm visits were done in The Netherlands, Switzerland and France in order to find out what genotypes are being used and how they perform. There are differences between countries, systems (organic vs free range) and groups of breeds. There is not just one genotype suitable for organic and free range systems.
\end{abstract}

Key words: laying hens, organic, free range, genotypes

\section{Introduction}

Due to increased mobility and reduced temperature control in free range and organic systems compared to cages and closed houses, free range and organic hens probably have a higher energy requirement (Ketelaars et al., 1985; Anderson, 2010). Testing of genotypes of hens that have to perform in free range and organic conditions is preferably done in free range and organic conditions. The aim of our study was to obtain information on how different genotypes or groups of genotypes of laying hens perform under organic and conventional free range conditions and if certain genotypes or groups of genotypes thus are more suitable for these systems. In the first stage the information was collected by an inquiry and in the second stage by farm visits among a part of the farms in the inquiry. During the visits we collect information on management practices and score the birds to get uniform information on physical condition of the hens. Here we present the results of the judgement of the birds.

\section{Material and methodology}

Switzerland, France and The Netherlands were selected as being representative for different European situations, because these countries were expected to differ with regard to general climate, farm size, preference for specific genotypes and housing systems. In each country totally approximately 100 organic and conventional free range farms were sampled from a national data base available to resp. FiBL for Switzerland, WUR-LR and Louis Bolk Institute for The Netherlands and ISA for France. An identical questionnaire was sent by (e-)mail to all farmers, but most questionnaires were completed by telephone interviews. Data collected on flock level comprised genotype, rearing system, specific treatments (e.g. beak treatment, vaccination, medication), feeding regimes (e.g. roughage, additional grain), and performance data (egg production per hen housed and mortality at 60 
week of age). Additional questions were on causes of mortality and feathering condition of the hens and estimates of the use of the outside run. The genotype was classified according to 'brand-name' i.e. specific cross. These crosses were later summarised in genetic groups: 'white' i.e. white hens laying white eggs, 'brown' i.e. brown hens laying brown eggs, 'silver' i.e. white hens laying brown eggs, or 'mixed' i.e. birds of different genotypes in one group. The results of this survey are described by Leenstra et al (2012).

From the 100 farms per country a subset was chosen for visits. The farms were visited when the hens were between 45 and 50 weeks old. Per farm 50 animals were caught, scored, marked and left free again. The animals were scored according to the protocol developed in the LayWel project (Tauson et al., 2005). According to this protocol neck, vent/cloaca, back, wings, tail, belly wounds, wounds on combs and feet pads were scored on a scale of 1(bad) to 4 (good). The keel bones

\section{Results of the enquiry}

The results of the enquiry are published in British Poultry Science and therefore will only be briefly discussed here. In Switzerland, France and The Netherlands in total 273 farmers with free ranging laying hens (organic and conventional), having 318 flocks with data for analysis, were interviewed. In total, almost 20 different genotypes (brands) or mixtures of genotypes were present on the farms. In France only 'brown' hens were housed. In Switzerland and The Netherlands there were 'brown', 'white' and 'silver' hens. In Switzerland also mixed flocks were present. The overall effect of system (organic vs. conventional) on egg production and mortality was significant, with higher mortality and lower egg production for organic flocks. In pair wise comparisons within country the difference was highly significant in The Netherlands and showed a non-significant tendency in the same direction in Switzerland and France. White hens tended to perform better than brown hens. Silver hens appeared to have a higher mortality and lower production per hen housed at 60 weeks of age. There were no significant correlations between production, mortality, feather condition and use of outside run on the one hand and flock size on the other. There was more variation in mortality and egg production among farms with a small flock size than among farms with a large flock size (Leenstra et al., 2012).

Table 1. Genotypes used on Dutch and Swiss organic and free range farms

\begin{tabular}{|c|c|c|c|c|c|}
\hline Genotype & Group & $\begin{array}{c}\text { NL } \\
\text { organic }\end{array}$ & $\begin{array}{c}\text { NL } \\
\text { free range }\end{array}$ & $\begin{array}{c}\mathrm{CH} \\
\text { organic }\end{array}$ & $\begin{array}{c}\mathrm{CH} \\
\text { free range }\end{array}$ \\
\hline Hyline Brown & Brown & & & & 1 \\
\hline H\&N Brown Nick & Brown & 1 & & 5 & 1 \\
\hline Lohmann Brown Lite & Brown & 6 & 6 & & \\
\hline Lohmann Brown Classic & Brown & 2 & 2 & 3 & 5 \\
\hline Lohmann Brown & Brown & 1 & & 1 & \\
\hline ISA Brown & Brown & 2 & 6 & & \\
\hline LSL & White & & 2 & & \\
\hline LSL Classic & White & & & 4 & 4 \\
\hline Hyline White & White & & & & 1 \\
\hline H\&N Super Nick & White & & & & 3 \\
\hline Dekalb Amberlink & Silver & 6 & & & \\
\hline Hyline Silver & Silver & 3 & & & \\
\hline Mixed & Mixed & 1 & & 10 & 7 \\
\hline To be confirmed & & & & 1 & \\
\hline Total nr of flocks & & 22 & 16 & 24 & 22 \\
\hline
\end{tabular}




\section{Results of the farm visits}

The farm visits are still being done, which means that here we can present only preliminary results from the visits until February 2012. In Switzerland 14 organic farms with 24 flocks and 5 free range farms with 22 flocks were visited. In The Netherlands 20 organic farms with 22 flocks and 13 free range farms with 16 flocks were visited. The farm visits took place between March 2011 and February 2012. Table 1 gives an overview of the genotypes and the type of farms where they are being kept.

Because we can only present preliminary results concerning the farm visits, we don't want to give results per genotype. Therefore in table 2 the results of the animal scorings* per country and system (organic or conventional free range) are presented.

\section{Table 2. Results of animal scorings on Dutch and Swiss organic and free range farms}

\begin{tabular}{|l|c|c|c|c|}
\hline & $\begin{array}{c}\text { Netherlands } \\
\text { organic }\end{array}$ & $\begin{array}{c}\text { Netherlands } \\
\text { free range }\end{array}$ & $\begin{array}{c}\text { Switzerland } \\
\text { organic }\end{array}$ & $\begin{array}{c}\text { Switzerland } \\
\text { free range }\end{array}$ \\
\hline Total nr of flocks & 22 & 16 & 24 & 22 \\
\hline Comb wounds & 3.1 & 3.4 & 3.4 & 3.3 \\
\hline Keel bone & 3.0 & 3.0 & 3.6 & 3.4 \\
\hline Belly wounds & 3.8 & 3.9 & 4.0 & 3.9 \\
\hline Foot pads & 3.4 & 3.4 & 3.2 & 3.1 \\
\hline Neck feathers & 3.4 & 2.7 & 3.8 & 3.4 \\
\hline Belly feathers & 2.9 & 3.1 & 3.7 & 3.6 \\
\hline Back feathers & 2.6 & 3.1 & 3.6 & 3.5 \\
\hline Tail feathers & 2.8 & 2.7 & 3.5 & 3.3 \\
\hline Wing feathers & 3.4 & 3.2 & 3.6 & 3.4 \\
\hline
\end{tabular}

$* 1=$ bad, 4 = good; average of all flocks scored, scoring 50 hens per flock

\section{Discussion about results of the enquiry}

As we aimed for a 50/50 distribution between conventional free range and organic farms, organic farms are overrepresented compared to the general situation in the 3 countries. The distribution of genotypes across countries and systems in the survey mirrors the general picture in the three countries: in France only brown hens and in Switzerland and The Netherlands more variation in types. In Switzerland the number of mixed flocks was high compared to the other countries. White hens are rather scarce in organic and free range systems, although less so in Switzerland than in The Netherlands. This might be due to the preference for brown eggs in the North-western part of Europe (Arthur and O'Sullivan, 2005), the main market for The Netherlands and/or the association among consumers between brown egg shells and free range systems and/or healthier eggs (Johnston et al., 2011). Silver hens were introduced some years ago as more suitable genotypes for non-cage housing systems, as they were expected to combine the advantages of the good feathering of a white hen with the desired brown egg shells. Today, they are mainly present in The Netherlands. The introduction of silver hens and the increase in free range and organic systems more or less went together. However, our results do not indicate that silver hens are specifically suitable genotypes for free range and organic systems. Our finding that mortality is on average higher in organic than in free range systems supports findings of Hovi et al. (2003), Zeltner and Maurer (2009), Lambton et al. (2010), and Anderson (2010). From our enquiry among farmers we could not get clear indications on causes of mortality. However, from another study among 30 Dutch organic laying hen farmers it seems that $50 \%$ of the dead animals had E. coli or chronic gut infections, $10-15 \%$ of the dead hens died because of smothering and other 'accidents' and in $25 \%$ of the cases the cause of death was not clear or other than mentioned above (J. Wagenaar, 2011, personal information). There was no relation between mortality and flock size, except that variation in mortality was higher among small flocks than among large flocks. 
Egg production per hen housed at 60 weeks of age is related to mortality. Differences in production between countries, systems and genotypes follow almost completely differences in mortality. While the number of flocks of white hens is rather low, their production in organic and free range systems is high compared to the other genotypes. Silver hens, in contrast, show relatively high mortality and consequently low production per hen housed. Genotype on the one hand and country and flock size on the other are to some extent confounded (more white hens and small flocks in Switzerland and more silver hens and large flocks in The Netherlands). In the enquiry white hens were reported to have a better feather cover than brown hens, while feather scores for silver hens were lowest. Despite the difference in combination of the grandparent lines, silvers apparently are not different from brown hens in feather quality.

\section{Discussion about the results of the farm visits}

Since our dataset is not complete yet and too small to do statistics on, we would not like to run the risk to draw wrong conclusions concerning characteristics per genotype, system or country. Therefore we have presented only the rough results that are available up until now.

\section{Conclusions from the enquiry}

- From the large number of different genotypes present on free range and organic farms we conclude that there is no preferred genotype for free range systems and that no genotype seems to be best suited for those conditions.

- Organic and free range farms housed a wide variety of genotypes in Switzerland and The Netherlands. In France only brown hens were housed, in Switzerland and The Netherlands brown, white and silver hens. In Switzerland also mixed flocks were present.

- There was a tendency for slightly higher mortality and lower egg production per hen housed at 60 weeks of age in organic flocks compared to conventional free range flocks in Switzerland and France and a significant difference in the same direction in The Netherlands.

- White hens tended to perform better than brown hens, while silver hens appeared to have a higher mortality and lower production per hen housed at 60 weeks of age compared to white and brown flocks.

- There were no significant correlations between production, mortality, feather condition and use of outside run on the one hand and flock size on the other.

- There was more variation in mortality and egg production among farms with a small flock size than among farms with a large flock size.

\section{Conclusions from the farm visits}

- Still a large number of genotypes is being kept, which supports the conclusion from the enquiry that there is no preferred genotype.

- In the Netherlands mainly brown and silver genotypes were kept. In Switzerland also white genotypes and mixed flocks were kept.

\section{Suggestions to tackle the future challenges of organic animal husbandry}

In order to guarantee an acceptable level of animal health and welfare, as well as an economically and environmentally sound organic animal husbandry, the best suitable genotypes or breeds should be used. To identify which genotypes are suitable, the performance of the available genotypes should be monitored on farms on a regular base. For egg production and mortality this might be possible through contributions of farmers in an (international) database. Professional control on 
such a database is in our opinion a necessary condition. To learn more about possible causes of differences in performance farm visits by experienced observers is required.

\section{References}

Anderson KE (2010) Range egg production; is it better than in cages? 2010 MPF Convention, March 16-18 2010, St Paul, Minnesota, USA, http://www.midwestpoultry.com

Arthur JA \& O'Sullivan N (2005) Breeding chickens to meet egg quality needs. International Hatchery Practice 19: 7-9.

Hovi M, Sundrum A \& Thamsborg SM (2003) Animal health and welfare in organic livestock production in Europe: current state and future challenges. Livestock Production Science, 80: 41-53.

Johnston NP, Jefferies LK, Rodriguez B \& Johnston DE (2011) Acceptance of brown-shelled eggs in a white-shelled egg market. Poultry Science 90: 1074-1079

Ketelaars HE, Arets A, Hel W Van de, Wilbrink AJ \& Verstegen WA (1985). Effect of housing systems on the energy balance of laying hens. Netherlands Journal of Agricultural Science 33: 35-43.

Lambton SL, Knowles TC, Yorke C \& Nicol CJ (2010) The risk factors affecting the development of gentle and severe feather pecking in loose housed laying hens. Applied Animal Behaviour Science, 123: 32-40.

Tauson R, Kjaer J, Maria GA, Cepero R and Holm KE (2005). Applied scoring of integument and health in laying hens. http://www.laywel.eu/web/pdf/deliverables\%2031-33\%20health.pdf

Wagenaar J (Louis Bolk Institute) Personal information 2011. Researcher animal health and welfare.

Zeltner E \& Maurer V (2009) Welfare of organic poultry. In: proceedings of WPSA Poultry Welfare Symposium, Italy, 18-22 May, 2009, 104-112.

\section{Acknowledgement}

The authors gratefully acknowledge funding from the European Commission, under the Seventh Framework Programme for Research and Technological Development, for the Collaborative Project Low Input Breeds (Grant agreement No 222623)The views expressed in this publication are the sole responsibility of the author(s) and do not necessarily reflect the views of the European Commission. Neither the European Commission nor any person acting on behalf of the Commission is responsible for the use which might be made of the information contained herein.

The Dutch contribution is partly funded by the Bioconnect program of the Dutch Ministry of Economics, Innovation and Agriculture.

We would also like to thank the farmers participating in the survey and the organizations helping with data collection. 